\title{
Neurosarcoidosis Presenting as Young-Onset Chronic Headache: A Case Report
}

\author{
Barun Kumar Ray ${ }^{a}$ Rahul Kumar Thakur ${ }^{b}$ Anish Kumar Shah ${ }^{c}$ \\ Biplov Adhikarid $^{d}$ Dipesh Kumar Mandal ${ }^{\mathrm{e}}$ Ranjit Pandey ${ }^{f}$
}

${ }^{a}$ Department of Internal Medicine, B.P. Koirala Institute of Health Sciences, Dharan, Nepal; bepartment of Internal Medicine, Jacobi Medical Center/Albert Einstein College of Medicine, New York, NY, USA; 'Department of Internal Medicine, Bronxcare Hospital, New York, NY, USA; dDepartment of Internal Medicine, Medstar Union Memorial Hospital, Baltimore, MD, USA; ' Department of Internal Medicine, Banaras Hindu University, Varanasi, India; fDepartment of Internal Medicine, Postgraduate Institute of Medical Education and Research (PGIMER), Chandigarh, India

\section{Keywords}

Neurosarcoidosis $\cdot$ Chronic headache $\cdot$ Meningeal tuberculosis $\cdot$ Facial nerve palsy

\begin{abstract}
Sarcoidosis causes granulomatous inflammation in multiple organ systems. Lungs are the most commonly affected organs. In 50\% of the cases with lung involvement, other organs are also involved. The central nervous system is one of the rarer organ systems involved - comprising $5-15 \%$ of all the cases. Neurological involvement, when present, can affect virtually every part of the central and peripheral nervous system. We present an interesting case of a 30 -year-old nonalcoholic, nonsmoker man who presented with a chronic headache of 6 years. He had a recent swaying of the body for 6 months and mild forgetfulness for 4 months. The patient had 2 episodes of facial nerve palsy in the past - which had responded to the treatment of Bell's palsy; however, the patient had some residual findings on presentation. While CSF findings suggested meningeal tuberculosis, the radiological evidence and biopsy confirmed the diagnosis of neurosarcoidosis. On follow-up after 6 weeks of steroid therapy, the patient displayed a marked improvement in headache, cognitive function, and a good response on imaging studies. Our case emphasizes that neurosarcoidosis should be considered in the differential diagnosis of chronic headaches and recurrent facial nerve palsy despite being very rare.
\end{abstract}


Ray et al.: Neurosarcoidosis Presenting as Young-Onset Chronic Headache

\section{Introduction}

Chronic headache is one of the most common medical complaints among people of all age groups. However, in the younger population, it can be highly debilitating as it can impact patients' quality of life, resulting in several physical and mental disorders [1]. Chronic headache secondary to neurosarcoidosis is often overlooked because of its rarity in occurrence [2]. It is difficult to diagnose neurosarcoidosis, particularly when other organ systems are not involved. Detailed examination and investigation can help rule out other mimicking conditions. In this case, the cerebrospinal fluid (CSF) analysis and radiological investigation were highly supportive of meningeal tuberculosis. But, failure to improve despite antitubercular treatment led to further investigation to diagnose the condition and significant impact on his further treatment.

This case report reviews a patient who presented with a chronic headache of 6 years and was later diagnosed with neurosarcoidosis with no symptoms suggestive of other systems involvement. Neurosarcoidosis is rare, and presentation with chronic headache is highly unusual. Therefore, this case points to the need of evaluating headaches among young individuals with full detail - keeping neurosarcoidosis as a possible differential in mind - along with adding to the list of few reported cases of neurosarcoidosis.

\section{Case Presentation}

An Asian male of 30 years presented to our outpatient department with a history of chronic headaches, swaying of the body, and forgetfulness. He complained of holocranial, throbbing, and moderate intensity, on and off headache for 6 years. The headache occurred 3-4 times a week, with each episode lasting 15-30 min. It was partially relieved on taking analgesics. He also complained of swaying while walking for the last 6 months and being forgetful for the previous 4 months. His past medical history was significant for an episode of presumed right-sided Bell's palsy 3 years back - treated with a short course of oral steroids for 1 week with marked improvement - and another bout of facial asymmetry in the left side 7 months before. For the latter episode, radiological evidence and CSF analysis suggested meningeal tuberculosis, and he was started on antitubercular therapy and steroids. The patient had symptomatic improvement with the treatment, but he defaulted on the treatment 2 months later. He was a nonsmoker, nonalcoholic, and did not have any significant medical and surgical illness. On his current visit, he denied any fever, weakness, and sensory or speech changes. His vitals were stable. On a neurological examination, his mini-mental state exam (MMSE) score was 21/30 with poor attention. He had signs suggestive of residual left lower motor neuron cranial nerve VII palsy. His pupils were bilaterally reactive to light and accommodation. He had a broad-based gait with swaying on both sides, bilateral dysmetria with left-sided predominance, and impaired rapid alternating movements with horizontal gaze-evoked nystagmus. His motor and sensory examinations were normal, with no signs of meningeal irritation. The rest of his systemic examinations were unremarkable.

His initial blood investigations - complete metabolic panel, hematological, human immunodeficiency virus, treponemal, tubercular, and immunological - were inconclusive. His lumbar puncture showed elevated white cell count with 80 lymphocytes, the CSF protein level was very high at $720 \mathrm{mg} / \mathrm{dL}$ (reference: $<60 \mathrm{mg} / \mathrm{dL}$ ), and sugar level was low at $26 \mathrm{mg} / \mathrm{dL}$ (reference: $50-80 \mathrm{mg} / \mathrm{dL}$ ). The adenosine deaminase level in CSF was also raised at $30 \mathrm{U} / \mathrm{L}$ (reference: $<10.5 \mathrm{U} / \mathrm{L}$ ); however, the CSF was negative for acid-fast bacilli.

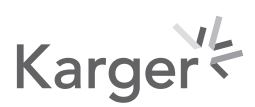



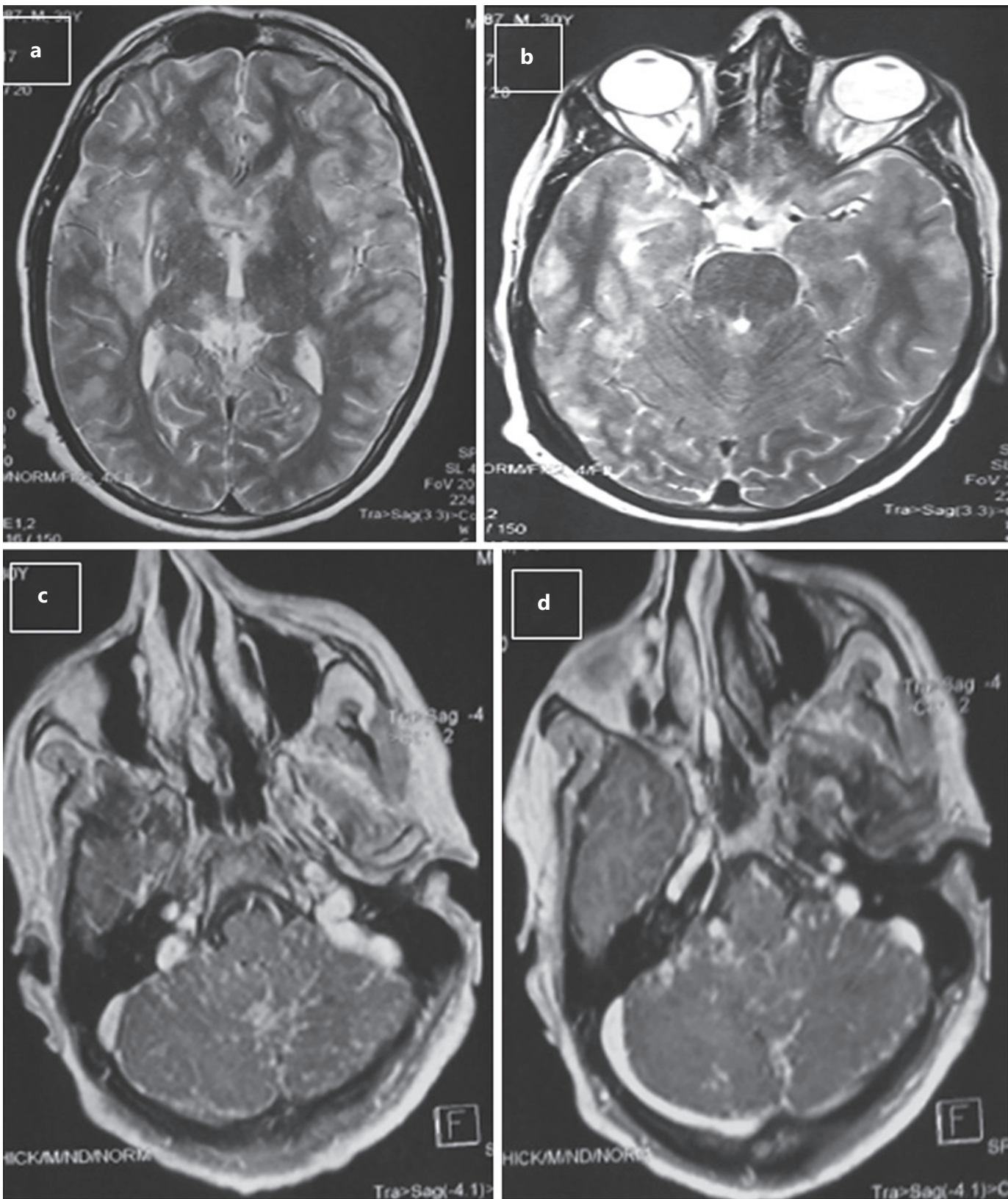

Fig. 1. The patient's MRI showing multiple hyperintensities in cerebral hemispheres predominantly in the right temporal lobe and hypothalamus with nodular leptomeningeal enhancement. a, b T2WI axial brain image. c, d Postcontrast T1WI axial brain image.

Subsequently, contrast-enhanced magnetic resonance imaging (MRI) of the head revealed diffuse nodular enhancing leptomeningeal and subpial enhancement involving both supra- and infratentorial compartments, spinal cord, and cauda equine (shown in Fig. 1). Additionally, there was multiple focal altered signal intensity in the bilateral cerebrum and cerebellum suggestive of tuberculosis/sarcoidosis.

Further investigations were carried out to confirm the diagnosis. Contrast-enhanced computed tomography chest and abdomen showed enlargement of the right paratracheal lymph nodes (largest $15 \times 9 \mathrm{~mm}$ ) and the right hilar lymph nodes (largest $16 \times 10 \mathrm{~mm}$ ). 

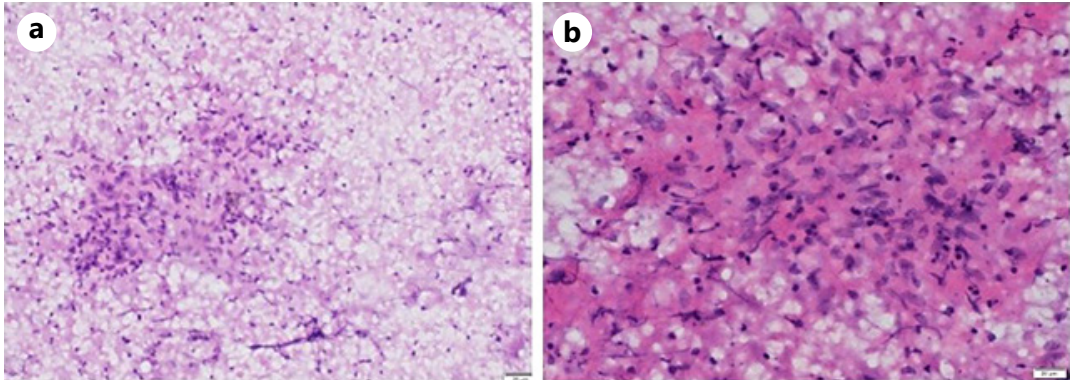

Fig. 2. Low-power (a) and high-power (b) histology showing granulomatous inflammation with noncaseating granuloma, suggestive of sarcoidosis.

Endobronchial ultrasound-guided transbronchial needle aspiration was done, which showed inflammation with noncaseating granuloma consistent with the diagnosis of sarcoidosis (shown in Fig. 2).

Steroids are the first-line treatment of neurosarcoidosis. If the patient deteriorates, does not respond, or has a contraindication to steroids, he/she is started on alternative therapies - immunomodulatory therapies like methotrexate, azathioprine, infliximab, etc. Once we established the diagnosis of the probable neurosarcoidosis in this patient, he was treated with high-dose oral prednisone at $1 \mathrm{mg} / \mathrm{kg} /$ day for 6 weeks, after which the dose was gradually tapered. During follow-up after 6 weeks, the patient reported marked improvement in his headache. His cognitive dysfunction also improved gradually, with a mini-mental state exam score of 25/30. Repeat MRI of the brain revealed a decrease in the leptomeningeal enhancement. Repeat contrast-enhanced computed tomography chest was advised to evaluate for pulmonary response to treatment, but the patient denied. He did not report any adverse effects of corticosteroid treatment on close observation during his follow-ups.

\section{Discussion}

Our case was unique as the patient presented with chronic headaches of 6 years, with intermittent facial nerve involvement. Considering that Southeast Asia is highly endemic for tuberculosis, and the laboratory findings of sarcoidosis resemble tuberculosis, it is usual and imperative to manage the patient as meningeal tuberculosis in this particular resource-limited setting [3]. A rare diagnosis like sarcoidosis is suspected after patients fail to improve on treatment to common conditions like tuberculosis. Sarcoidosis causes granulomatous inflammation in multiple organ systems. Lungs are the most commonly affected organs. In $50 \%$ of the cases with lung involvement, other organs are involved, while isolated extrapulmonary involvement is seen in $2 \%$ of all the cases [4]. The central nervous system is one of the rarer organ systems to be involved, comprising $5-15 \%$ of all the patients [5].

Clinical presentation of neurosarcoidosis varies widely and depends upon the part of the nervous system involved. It can involve any part such as cranial nerves, brain, spinal cord, meninges, and peripheral nerves [6]. Cranial neuropathy is the most common presentation of neurosarcoidosis. Involvement of all the cranial nerves has been reported; however, facial nerve palsy is present in about $25-50 \%$ of patients [7]. Other presentations include meningitis, loss of consciousness, seizures, headaches, and peripheral neuropathy. It is noteworthy that our patient presented with a relatively rare symptom 
of chronic headache with 2 episodes of facial nerve palsy in the past. This likely results from the nerve granulomas increased intracranial pressure or granulomatous basal meningitis [8]. This presentation in the appropriate clinical context should raise the suspicion of neurosarcoidosis.

In addition to the rarity of neurosarcoidosis, the absence of firm diagnostic criteria and unavailability of the tissue biopsy sometime present challenges in making the diagnosis. The neuroimaging findings complemented with the CSF findings can help exclude differentials and aid in the diagnosis $[9,10]$. The imaging modality of choice for the neurological disease is contrast-enhanced MRI. Leptomeningeal involvement presents as a diffuse or nodular thickening and enhancement on contrast-enhanced T1-weighted images, a pattern that is commonly observed in infectious (tuberculosis) and lymphomatous meningitis [11]. CSF analysis is routinely done, showing inflammatory changes, with mononuclear cell pleocytosis, high protein content, and low to normal glucose. Imaging and CSF findings in neurosarcoidosis resemble meningeal tuberculosis; therefore, in regions with a high burden of tuberculosis, diagnosis of neurosarcoidosis is usually delayed, particularly in absence of other systemic findings.

Despite the dilemma in diagnosis, it is important to be aware of neurosarcoidosis in younger patients with headaches and recurrent focal neurological presentations. It is because it demands different treatment compared to infective processes. Although a randomized control trial to look for definitive treatment of neurosarcoidosis has not been done, the treatment of neurosarcoidosis is mainly derived from expert opinions and available retrospective studies. Three tiers of treatments have been defined in the medical literature based on the intensity of therapy required, which in turn depends on the presenting symptoms of the patients [12]. Mild symptoms like isolated facial nerve palsy can be treated with short courses of steroids, while disease with spinal cord involvement often needs second-line treatment - immunosuppressive therapy - or third-line treatment - monoclonal antibodies $[13,14]$. Glucocorticoid is still considered as the first line for treatment of neurosarcoidosis [15].

Neurosarcoidosis, a rare diagnosis, can be mistaken for tuberculosis in resource-limited settings. A prudent clinician should be mindful of neurosarcoidosis as a differential while considering chronic neurological symptoms like headache, cranial nerve palsy, and disorganized body movements. As highlighted in our case report, early recognition and prompt treatment with steroids can help alleviate patients' symptoms.

\section{Statement of Ethics}

Since this study is a case report, it is exempt from ethics committee approval. We have obtained written informed consent from the patient for publication of this case report and any accompanying images.

\section{Conflict of Interest Statement}

The authors declare that they have no competing interests.

\section{Funding Sources}

None of the authors have received any financial assistance for this manuscript.

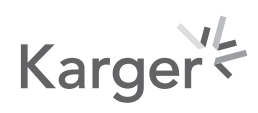


Ray et al.: Neurosarcoidosis Presenting as Young-Onset Chronic Headache

\section{Author Contributions}

B.K.R., R.K.T., and A.K.S. contributed to the concept of the manuscript and drafted the manuscript. B.A., D.K.M., and R.P. revised the manuscript and contributed to the conception of the manuscript. B.K.R., R.K.T., A.K.S., D.K.M., B.A., and R.P. contributed to the obtaining and interpreting of the clinical information. All authors read and approved the final version of the manuscript.

\section{Data Availability Statement}

All data related to this case report are contained within the manuscript.

\section{References}

1 Langeveld JH, Koot HM, Loonen MC, Hazebroek-Kampschreur AA, Passchier J. A quality of life instrument for adolescents with chronic headache. Cephalalgia. 1996 May;16(3):183-96; discussion 137.

2 Vukojevic Z. EHMTI-0060. Neurosarcoidosis and chronic headache-case report. J Headache Pain. 2014 Dec; 15(1):1.

3 Jarman PR. Meningeal granulomas: sarcoidosis or tuberculosis? Differentiation can be difficult. BMJ. $1995 \mathrm{Feb}$ 25;310(6978):517-20.

4 Baughman RP, Teirstein AS, Judson MA, Rossman MD, Yeager H Jr, Bresnitz EA, et al. Clinical characteristics of patients in a case control study of sarcoidosis. Am J Respir Crit Care Med. 2001 Nov 15;164(10 Pt 1):1885-9.

5 Dias R, Ferreira IH, Faria R. Viscous leptomeningeal pseudotumoural masses and multiple cranial neuropathy - severe presentation of neurosarcoidosis. Eur J Case Rep Intern Med. 2020;7(3):001453.

6 Stern BJ, Royal W 3rd, Gelfand JM, Clifford DB, Tavee J, Pawate S, et al. Definition and consensus diagnostic criteria for neurosarcoidosis: from the neurosarcoidosis consortium consensus group. JAMA Neurol. 2018 Dec 1;75(12):1546-53.

7 Stern BJ, Krumholz A, Johns C, Scott P, Nissim J. Sarcoidosis and its neurological manifestations. Arch Neurol. 1985 Sep 1;42(9):909-17.

8 Hoitsma E, Faber CG, Drent M, Sharma OP. Neurosarcoidosis: a clinical dilemma. Lancet Neurol. 2004 Jul 1; 3(7):397-407.

9 Zajicek JP, Scolding NJ, Foster O, Rovaris M, Evanson J, Moseley IF, et al. Central nervous system sarcoidosis: diagnosis and management. QJM. 1999 Feb 1;92(2):103-17.

10 Feizi P, Tandon M, Khan E, Subedi R, Prasad A, Chowdhary A, et al. Overcoming the elusiveness of neurosarcoidosis: learning from five complex cases. Neurol Int. 2021 Jun;13(2):130-42.

11 Ginat DT, Dhillon G, Almast J. Magnetic resonance imaging of neurosarcoidosis. J Clin Imaging Sci. 2011;1:15.

12 James WE, Baughman R. Treatment of sarcoidosis: grading the evidence. Expert Rev Clin Pharmacol. 2018 Jul 3;11(7):677-87.

13 Lower EE, Broderick JP, Brott TG, Baughman RP. Diagnosis and management of neurological sarcoidosis. Arch Intern Med. 1997 Sep 8;157(16):1864-8.

14 Bradley DA, Lower EE, Baughman RP. Diagnosis and management of spinal cord sarcoidosis. Sarcoidosis Vasc Diffuse Lung Dis. 2006 Mar 1;23(1):58-65.

15 Voortman M, Drent M, Baughman RP. Management of neurosarcoidosis: a clinical challenge. Curr Opin Neurol. 2019 Jun;32(3):475-83. 